\title{
Dilated Cardiomyopathy Associated with Celiac Disease: A Case Report
}

\author{
Salma Elnour ${ }^{1}$, Maram Hashim ${ }^{1}$, and Halah Ibrahim ${ }^{1}$ \\ ${ }^{1}$ Shaikh Khalifa Medical City
}

June 9, 2021

\begin{abstract}
Celiac disease is a common immune-mediated inflammatory disease of the small intestine caused by sensitivity to gluten. It's commonly associated with various autoimmune disease, but rarely with cardiomyopathy. Our case describes a 33 year old woman presented with exertional dyspnea and fatigue, found to have celiac disease and dilated cardiomyopathy.
\end{abstract}

Title: Dilated Cardiomyopathy Associated with Celiac Disease: A Case Report

Authors :Salma Elnour, Maram Hashim, Halah Ibrahim

Authors' affiliations : Department of Internal Medicine, Sheikh khalifa Medical City, Al karamah Street, Abu Dhabi, United Arab Emirates.

\section{Corresponding author}

Salma Elnour

Sheikh khalifa Medical City

PO BOX 48793

Abu Dhabi, United Arab Emirates

Sanour@seha.ae

\section{Key clinical message}

Celiac disease is an autoimmune disease that is associated with different conditions. cardiomyopathy associated with celiac disease needs high degree of clinical suspicion to make the diagnosis, as a gluten-free diet can improve heart function.

\section{Abstract}

Background: Celiac disease, an immune-mediated inflammation of the small intestine, occurs in genetically predisposed individuals, and is caused by sensitivity to dietary gluten and related proteins. The global prevalence is approximately $1 \%$. Celiac disease is frequently associated with extra-intestinal manifestations, including iron deficiency anemia, dermatologic eruptions, diabetes mellitus, thyroid disease, and connective tissue disorders, but is rarely associated with cardiomyopathy. Case presentation: Our case describes a 33 year old women, who presented with exertional dyspnea and fatigue, and found to have severe iron deficiency anemia and dilated cardiomyopathy, secondary to celiac disease. Conclusion: High clinical suspicion is needed to identify celiac disease in patients diagnosed with dilated cardiomyopathy, as an improvement in cardiac function has been reported with strict adherence to a gluten free diet. 


\section{Key Words}

Case report, Celiac disease, Iron deficiency anemia, Hypothyroidism, Dilated cardiomyopathy, Gluten free diet

\section{Background}

Celiac disease (CD), an immune-mediated inflammation of the small intestine, occurs in genetically predisposed individuals, and is caused by sensitivity to dietary gluten and related proteins [1]. Globally, the prevalence of celiac disease is $1.4 \%$ based on serologic test results, and $0.7 \%$ based on biopsy results [2]. Celiac disease is frequently associated with extra-intestinal manifestations, including iron deficiency anemia, dermatologic eruptions, diabetes mellitus, thyroid disease, and various connective tissue disorders, but is rarely associated with cardiomyopathy [3].

\section{Case Presentation}

A 33 year old Ethiopian woman presented to the emergency department after referral from her dentist for "looking pale." She reported a 2-month history of exertional dyspnea and fatigue. She denied chest pain, shortness of breath at rest, palpitations or orthopnea. There was no history of abnormal bleeding or bruising, heavy menses, melena or hematochezia. The patient denied cough, fever, abdominal pain, diarrhea or urinary complaints. She denied any rashes or weight loss.

The patient was employed as a housemaid. She had no past medical or surgical history and no previous hospitalizations. There was no family history of chronic or autoimmune diseases. She denied taking any prescription or over the counter medication. She denied alcohol or illicit drug use and was a non-smoker. The patient also denied sick contacts or recent travel.

On initial examination, she appeared pale but otherwise healthy. Her body mass index was 20.5. She was afebrile with a temperature of $37^{\circ} \mathrm{C}$, blood pressure $124 / 74$ without orthostatic changes, heart rate of 88 bpm, and oxygen saturation of $100 \%$ on room air. Neck examination revealed a non-tender diffuse goiter, no lymphadenopathy, and jugular venous pressure was not elevated. Cardiovascular examination revealed a grade $3 / 6$ pan systolic murmur, maximum at the apex with radiation to the axilla. Chest exam was clear on auscultation, with no peripheral edema. Abdomen was soft, non-tender with no organomegaly. Joint examination was unremarkable. There were no mucosal ulcers or skin rashes.

Initial labs revealed a microcytic anemia with hemoglobin of $32 \mathrm{~g} / \mathrm{dL}$ (117-155 g/dL), MCV $49.7 \mathrm{fL}$ (81-100 fL), $\mathrm{MCH} 11.1 \mathrm{pg}(27-34 \mathrm{pg})$, with WBC count 5.5x10^9/L (4.5-11x10^9/L), and platelets $394 \times 10^{\wedge} 9 / \mathrm{L}(140-$ $\left.400 \times 10^{\wedge} 9 / \mathrm{L}\right)$. Liver and kidney function tests were normal. Iron studies revealed iron level $1.4 \mathrm{mmol} / \mathrm{L}$ (5.8-34.5 mmol/L), transferrin $3.7 \mathrm{~g} / \mathrm{L}(2-3.6 \mathrm{~g} / \mathrm{L})$, transferrin saturation 0.02 (0.07-0.42), and ferritin 6 $\mathrm{mcg} / \mathrm{L}(15-150 \mathrm{mcg} / \mathrm{L})$. Vitamin B 12 level was 272pmol/L $(128-648 \mathrm{pmol} / \mathrm{L})$, and folate level was 22.4 $\mathrm{nmol} / \mathrm{L}(10.9-84.5 \mathrm{nmol} / \mathrm{L})$. Hemolysis panel was unremarkable with an LDH of $162 \mathrm{IU} / \mathrm{L}$ (135-214 IU/L) and haptoglobin of $0.64 \mathrm{~g} / \mathrm{L}(0.3-2 \mathrm{~g} / \mathrm{L})$.

ECG showed sinus rhythm with left bundle branch block. Serial troponin levels were normal. An echocardiography was performed, which showed a severely dilated left ventricle and severely reduced left ventricular systolic function, with left ventricular ejection fraction of 15-20\%. There was severe global hypokinesis of the left ventricle and grade II left ventricle diastolic dysfunction. Doppler samples suggested elevated left ventricular filling pressure. There was mild to moderate mitral regurgitation. Pulmonary artery systolic pressure was normal at $30-35 \mathrm{mmHg}$. A small pericardial effusion was noted.

The patient was admitted to the general medical ward for severe symptomatic iron deficiency anemia. She received 4 units of cross-matched red blood cells. Her hemoglobin subsequently increased to $96 \mathrm{~g} / \mathrm{L}$. Further work up showed anti-gliadin IgA $379.6 \mathrm{cu}$ (normal <19.9 cu), anti gliadin IgG $708.4 \mathrm{cu}$ (normal < 19.9 $\mathrm{cu}$ ), anti tissue transglutaminase IgA $3671.9 \mathrm{cu}$ (normal $<19.9 \mathrm{cu}$ ), anti-tissue transglutaminase IgG 2356.4 cu (normal < $19.9 \mathrm{cu})$. She underwent an upper endoscopy, which revealed normal mucosa. A duodenal biopsy showed total villous atrophy, crypt hyperplasia and intraepithelial lymphocytosis consistent with 
celiac disease. No granulomas were seen. The dietician was consulted and the patient was started on a gluten free diet.

CT cardiac coronaries was performed and revealed a dilated left ventricle and left atrium, absent coronary calcification with no evidence of coronary anomaly or atheromatous disease. Cardiac MRI showed dilated cardiomyopathy with severely dilated left ventricle, severely reduced left ventricular ejection fraction of $26 \%$, increased indexed LV mass and mid septal wall enhancement with small pericardial effusion. The patient was started on a medication regimen that was financially feasible, which included valsartan, bisoprolol, Ivarbadine and spironolactone.

To work up the goiter, thyroid function tests were done and showed TSH 22.4 milli $(0.27-4.2$ milli) and T4 7pmol/L (12-22 pmol/L), with elevated thyroid autoantibodies TPO Ab $44 \mathrm{IU} / \mathrm{ml}$ (normal $<34 \mathrm{IU} / \mathrm{ml}$ ), Thyroglobulin $\mathrm{Ab} 1816 \mathrm{IU} / \mathrm{ml}$ (normal $<115 \mathrm{IU} / \mathrm{ml}$ ). Other autoimmune work up was performed and showed low C3 $0.77 \mathrm{~g} / \mathrm{L}(0.9-1.8 \mathrm{~g} / \mathrm{L})$, normal C4, elevated rheumatoid factor of $64 \mathrm{IU} / \mathrm{ml}$ (normal $<14 \mathrm{IU} / \mathrm{ml}$ ). Antinuclear antibody and double stranded DNA were negative. Ultrasound of the thyroid revealed diffuse thyromegaly with increased vascularity. The patient was started on levothyroxine supplementation.

On discharge, the patient was doing well with no symptoms of heart failure. Her hemoglobin remained stable after transfusion. The patient was educated about celiac disease and the importance of a strict gluten free diet and scheduled for outpatient follow up.

\section{Discussion:}

This case of severe anemia and cardiomyopathy, without gastrointestinal symptoms, highlights the extraintestinal findings that should raise a high clinical suspicion for celiac disease. Celiac disease has a wide spectrum of clinical presentations, with both gastroenterological and extra-intestinal manifestations. Different clinical categories of $\mathrm{CD}$ have been described in literature, ranging from silent $\mathrm{CD}$, which is generally asymptomatic, to classic or typical CD, which is characterized by intestinal symptoms, and atypical or subclinical CD, which also includes extra-intestinal symptoms [1]. As the duodenum is the site of iron absorption and the major site of inflammation in patients with CD, iron deficiency anemia (IDA) is one of the most common clinical manifestations of $\mathrm{CD}$, and is present in over half of patients at the time of diagnosis $[1,2]$. IDA can be the only sign of CD, particularly in patients with atypical CD. Some studies have suggested that the degree of villous atrophy correlates with anemia severity [4]. For example, in a study of 405 adult celiac patients, Harper et al. documented a significantly higher prevalence of IDA (34\%) in patients with subtotal/total villous atrophy, when compared with patients with partial villous atrophy (13\%; p > 0.001)[5]. Annibale et al. also found a significant inverse correlation between hemoglobin concentration and the pathologic severity of duodenal biopsies in patients with CD [4].

Recent studies, with advanced diagnostic cardiac imaging, have highlighted the relationship between CD and cardiovascular diseases. Severely dilated left ventricle, left ventricular dysfunction, very low ejection fraction, pulmonary hemosiderosis, and heart block have all been reported in cardiomyopathy patients with CD [6]. Several mechanisms have been proposed to explain the etiology and progression of cardiomyopathy in celiac disease. Firstly, severe nutritional deficiencies due to chronic malabsorption can cause cardiomyopathy [3]. It has also been suggested that derangements in intestinal permeability in patients with CD may allow the absorption of luminal antigens or infectious agents and lead to myocardial damage through immunemediated mechanisms [3]. Finally, direct myocardial injury may result from an immune response against an antigen present in both the myocardium and the small intestine [3]. Understanding the relationship between celiac disease and cardiomyopathy can help explain the effects of a gluten -free diet in patients with cardiac manifestations. One case series described the effect of a gluten-free diet on cardiac performance in three patients with idiopathic dilated cardiomyopathy and celiac disease. In the two patients that strictly observed the gluten-free diet, a 28-month follow-up showed an improvement in echocardiographic parameters and quality of life measures. The third patient did not observe the gluten-free diet and presented with worsening echocardiographic parameters and cardiologic symptoms, and required additional medication therapy [7]. These data suggest that a gluten-free diet may have a significant beneficial effect on cardiac performance in 
patients with $\mathrm{CD}$ and idiopathic dilated cardiomyopathy [7].

Autoimmune disease is also strongly associated with CD, with an approximate prevalence of $20 \%$ in adults [8]. Hypothyroidism is the most common autoimmune manifestation, and occurs in $5 \%-15 \%$ of patients with $\mathrm{CD}$ [8]. Although the mechanism underlying the correlation between CD and hypothyroidism is unknown, the association is believed to be independent of gluten exposure, and is most likely related to a common genetic predisposition [9].

\section{Conclusion}

Our case highlights some of the conditions associated with celiac disease. These include iron deficiency anemia, dilated cardiomyopathy, and hypothyroidism. Cardiomyopathy associated with celiac disease is a serious and life-threatening condition if left untreated. A high degree of clinical suspicion is required to make a diagnosis. In patients with dilated cardiomyopathy and iron deficiency anemia, screening for CD is important, as a gluten-free diet can improve heart function.

\section{Conflict of interest}

The authors declare no conflicts of interest.

\section{Funding}

No funding was obtained for this study.

\section{Consent for publication}

Written consent was obtained from the patient.

\section{Ethical approval}

Ethical approval is not required at our institution to publish an anonymous case report.

Data sharing not applicable to this article as no datasets were generated or analysed during the current study

\section{References}

- Parzanese I, Qehajaj D, Patrinicola F, Aralica M, Chiriva-Internati M, Stifter S, Elli L, Grizzi F. Celiac disease: From pathophysiology to treatment. World J Gastrointest Pathophysiol. 2017 May 15;8(2):27-38. doi: 10.4291/wjgp.v8.i2.27.

- Singh P, Arora A, Strand TA, Leffler DA, Catassi C, Green PH, Kelly CP, Ahuja V, Makharia GK. Global Prevalence of Celiac Disease: Systematic Review and Meta-analysis. Clin Gastroenterol Hepatol. 2018 Jun;16(6):823-836.e2. doi: 10.1016/j.cgh.2017.06.037.

- Goel NK, McBane RD, Kamath PS. Cardiomyopathy associated with celiac disease. Mayo Clin Proc. 2005 May;80(5):674-6. doi: 10.4065/80.5.674.

- Stefanelli G, Viscido A, Longo S, Magistroni M, Latella G. Persistent Iron Deficiency Anemia in Patients with Celiac Disease Despite a Gluten-Free Diet. Nutrients. 2020 Jul 22;12(8):2176. doi: $10.3390 /$ nu12082176.

- Bhadada SK, Rastogi A, Agarwal A, Kochhar R, Kochhar R, Bhansali A. Comparative study of clinical features of patients with celiac disease \& those with concurrent celiac disease \& type 1 diabetes mellitus. Indian J Med Res. 2017 Mar;145(3):334-338. doi: 10.4103/ijmr.IJMR_666_14.

- Ciaccio EJ, Lewis SK, Biviano AB, Iyer V, Garan H, Green PH. Cardiovascular involvement in celiac disease. World J Cardiol. 2017 Aug 26;9(8):652-666. doi: 10.4330/wjc.v9.i8.652.

- Curione M, Barbato M, Viola F, Francia P, De Biase L, Cucchiara S. Idiopathic dilated cardiomyopathy associated with coeliac disease: the effect of a gluten-free diet on cardiac performance. Dig Liver Dis. 2002 Dec;34(12):866-9. doi: 10.1016/s1590-8658(02)80258-4.

- Qayed E, Shahnavaz N. Sleisenger and Fordtrans gastrointestinal and liver disease. Amsterdam: Elsevier; 2021. 
- Collins D, Wilcox R, Nathan M, Zubarik R. Celiac disease and hypothyroidism. Am J Med. 2012 Mar;125(3):278-82. doi: 10.1016/j.amjmed.2011.09.003. 